\title{
A Ciência como Cultura do Mundo Contemporâneo: a utopia dos saberes das (bio)ciências e a construção midiática do imaginário social ${ }^{1}$
}

MADEL LUZ" GESAR SABINO", RAFAEL S. MATTOS"

\section{Resumo}

O artigo trata essencialmente do papel das biociências, visto como uma cultura específica, no imaginário social contemporâneo, no que concerne à vida, à saúde e ao viver humano em todas as suas fases. Os meios de comunicação, sobretudo através da imprensa de divulgação de massa - sendo privilegiada no artigo a imprensa escrita - difundem para o conjunto da sociedade modos de pensar e agir derivadas das atividades científicas, em andamento ou finalizadas, na área das biociências, que compõem um campo disciplinar especializado de amplo espectro. Destacamos no texto a autoridade não apenas intelectual como moral do discurso normativo oriundo dessas atividades, face a outros discursos presentes na cultura, sejam eles tradicionais - de origem nativa ou externa, como as orientais - sejam eles paralelos atuais, como os das chamadas medicinas ou saberes terapêuticos alternativos derivados dos movimentos de contracultura que remontam aos anos setentas.

\footnotetext{
${ }^{1}$ Artigo elaborado a partir de Comunicação ao Congresso da ALAS de 2009, Buenos Aires, U.B.A. 6 p. Um projeto de pesquisa sobre imaginário cultural, ciência e mídia impressa foi elaborado sobre o tema (LUZ, SABINO, et. al, 2011, 25p.), entrando ainda em fase inicial, credenciado na UFRGS.

*Pós-Doutora em Saúde Coletiva. Professora colaboradora do Programa de Pós-Graduação em Saúde Coletiva da Universidade Federal Fluminense -UFF. (Brasil) E-mail: madelluz@uol.com.br ** Pós-Doutor em Saúde Coletiva. Professor Adjunto de Sociologia do Departamento de Estudos Políticos do Centro de Ciências Jurídicas e Políticas da Universidade Federal do Estado do Rio de Janeiro. DESP/CCJP/ UNIRIO. (Brasil). E-mail: cesarsabino@hotmail.com

*** Doutor em Saúde Coletiva. Professor Adjunto do Departamento de Ciências da Atividade Física do Instituto de Educação Física e Desportos da Universidade do Estado do Rio de Janeiro. (Brasil) E-mail: rafaelsmattos@uol.com.br
} 
Nossa hipótese é que esta influência normativa atinge áreas do viver e setores cada vez mais amplos das populações, sendo possível afirmar que as ciências sociais, sobretudo a sociologia, não vêm atribuindo à questão da vida e da saúde humanas a mesma importância que atribui a outros aspectos da vida social, e que é urgente pensar a cultura da vida e do viver veiculada pelas biociências.

Palavras chave: Biociências. Cultura. Imaginário social. Vida e saúde.

\section{Science as Culture in the Modern World: the utopia of kno- wledge in (bio)sciences and the media construction of social imaginary}

\section{Abstract}

This article deals with the growing influence of the so called biosciences, a large scope of disciplines concerning life, mainly human life, its origin, "normal" characteristics and evolution processes, from birth to death, in present culture. We are particularly interested in the effects of the discoveries and controversies of the diffusion of results of the research process in social imaginary. Through the mass media divulgation -specially press, mainly the magazines having as its objective the scientific research diffusion, an ensemble of rules about the way of conducting "normal" live all along its phases is communicated to the society as a whole. Our main questions are about to the influence of scientific culture, and the social effects of the normative nature of this kind of knowledge in present days. The scientific science, particularly the biosciences, has not only an intellectual power over public opinion and the other forms of knowledge present in contemporary society, as a moral power of conviction. People are convinced to behave as the bio scientific discourses proposes or their lives will be in danger, by their own fault. Respect to other forms of knowledge existing in present culture, no dialogue is actively proposed unless these forms show some "scientific evidences", which can demonstrate their "veracity" . We are worried about the dialogue capacity of this kind of producing facts and its verification, with traditional ways of knowing and conduction life, not only the ancient or oriental ways, but also the popular and alternative forms of knowing and conducting life in occidental contemporary culture since the seventies of last century.

Keywords: Biosciences. Culture. Social imaginary. Life and health. 


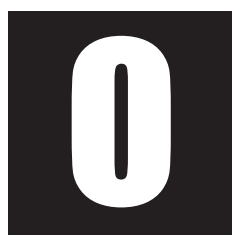

objetivo central deste texto é levantar questões, da perspectiva da sociologia política, em interface com a sociologia da comunicação e a comunicação social, sobre o papel que a ciência, definida como modo de produção de conhecimento baseado em observação sistemática ${ }^{2}$ desenvolvido no ocidente partir do século XVI (Luz, 1988, 2005, 2007, 2008), tem desempenhado na construção da cultura contemporânea, isto é, na geração de seu universo simbólico e, de fato, sobre o papel que desempenha no imaginário social que acompanha essa geração.

Quando nos referimos à categoria cultura, estamos nos reportando, em termos clássicos antropológicos, à dimensão irredutível da vida social onde são gerados e conservados - ou transformados - os sentidos atribuídos coletivamente pelos seres humanos aos seus modos fundamentais de agir, de pensar, de sentir e de relacionar-se em seu mundo, bem como às relações que estabelecem com o mundo dos "não humanos" (animais, vegetais, seres vivos ou inanimados, aos quais atribuem significados: sagrados, profanos, mágicos ou utilitários), distribuindo funções e organizando relações entre os mesmos.

Se preferíssemos adotar, neste artigo, ao invés de uma perspectiva sociopolítica, um viés antropológico, estaríamos nos referindo à dimensão simbólica da organização social. Nesta dimensão enraízam-se, nem sempre conscientemente, expressões complexas de representações de grupos e coletividades - mitos, lendas, concepções e ideologias - num crescendo de racionalização, assim como de instituições que lhes dão forma e orga-

\footnotetext{
${ }^{2}$ Outro modo de definir esta forma de conhecimento seria inserindo-a, como saber específico, em um universo epistêmico singular, desenvolvido em uma cultura histórica específica, como a episteme moderna clássica, como o fez Michel Foucault em sua obra Les Mots et les Choses (Foucault, 1965). Nossa abordagem se situa, entretanto, na interface da sociologia da cultura, do conhecimento, da comunicação e da política, enquanto a de Foucault situa-se nas fronteiras da epistemologia, da história e da antropologia dos saberes, em abordagem arqueológica (mais tarde, por influência de Nietzsche, em perspectiva genealógica).
} 
nização material sistemática, embasadas tanto nas representações como nas ações coletivas, dando sustentação não apenas a mentalidades, mas também a concepções e ideologias sociais, fornecendo assim suporte de legitimidade, ou pelo menos de reprodução continuada às sociedades, em sua diversidade, variedade e duração históricas (Geertz, 1977; Wagner, 1981; Cuche, 1998; Boas, 2005; Lévi-Strauss, 2008).

Tais instituições não são necessariamente sagradas ou religiosas. Tomemos como exemplo o mercado, no caso das sociedades modernas, que busca ser movido por decisões e ações racionais pragmáticas. No mesmo sentido, tomemos como exemplo as políticas públicas, econômicas ou sociais contemporâneas, que atingiram uma escala crescente de racionalização no último século, e que impregnam, inegavelmente, o imaginário presente, tanto em termos individuais como coletivos. Todas buscam a racionalidade (Javeau, 2005, p. 29-30). Sendo expressas por conjuntos de práticas discursivas e morais as quais pretendem completa objetividade, tanto por seu conteúdo racional - formulado em linguagem que busca a universalidade geralmente por meio de formalização matemática, obtida através de avaliações quantitativas estatísticas - como pelos dados empíricos em que se embasam, obtêm o consenso social - logo, a hegemonia para as mesmas - ajudando a manter a continuidade da ordem social para a qual produzem suas práticas discursivas (Nietzsche, 1978; Weber, 1984; 2004; Lévi-Strauss, 2002; Latour, 2009).

Habitualmente, em sociologia da cultura, termos relativos à dimensão simbólica, como sentidos, imaginário, representações sociais ${ }^{3}$, e até mesmo

\footnotetext{
${ }^{3}$ Segundo Durkheim (1972, p. 4), criador do termo: [...] maneiras de pensar, de agir e de sentir exteriores ao indivíduo, dotadas de um poder de coerção em virtude do qual se lhe impõem. Ou, prosseguindo: Representações sociais designam a camada mais antiga, e também a mais estável e a mais implícita da visão de mundo dos indivíduos. Nas representações sociais encontram-se categorias de classificação, imagens e símbolos que organizam as relações dos indivíduos entre si e com a natureza. Essa visão de mundo apresenta-se como natural não exigindo qualquer justificativa. (Bozon, 1995, p. 123-24). Ainda: [...] são esquemas de pensamento
} 
significados, costumam restringir-se à análise de ações sociais e de interações em coletividades culturais ou grupos de agentes sociais específicos, limitandose a pesquisas conduzidas por um olhar antropológico ou sociológico oriundo de abordagens interacionistas ou fenomenológicas que, nos anos $1970 \mathrm{~s}$ e 1980s, ganharam expressão nesta área do campo disciplinar das ciências humanas, em função de crise paradigmática nas ciências sociais.

Com efeito, os anos setenta e oitenta assinalaram, na história do pensamento social, uma forte e crescente polarização entre dois paradigmas metodológicos clássicos: o dito estrutural (correntes: funcionalista, estruturalista, marxista), isto é, aquele lidando com explicações e interpretações centradas na noção de totalidade ('estrutura social') como fundamento determinante da vida social em todos os seus aspectos: da organização social, das relações sociais, das ações coletivas e individuais, das motivações dos atores (ou sujeitos); e, em sentido paradigmático oposto, aquele dito acionalista (correntes: análise institucional, micro sociologia clássica, hermenêutica), isto é, centrado na interpretação das ações dos sujeitos como geradoras de sentidos, de valores e da própria ordem social, ações geralmente analisadas em termos das regras e normas de ordenação (considerando sua simetria ou assimetria) das interações entre os sujeitos, sua variabilidade no tempo e no espaço cultural, priorizando na análise sua mutabilidade, e não sua estabilidade.

Se a década de 70 pode ser vista, em termos gerais, como dominantemente estruturalista, a década de 80 é, com o ocaso das análises marxistas e funcionalistas e com a crescente dominância de estudos fenomenológicos e hermenêuticos, nitidamente acionalista nas ciências sociais

impensados que sob forma de um conjunto de pares de oposição binária [p. ex., forte/fraco, alto/baixo, bom/ruim, masculino/feminino, etc.,] funcionando como categorias de percepção, constroem as relações de poder do ponto de vista daqueles que afirmam sua dominação, fazendo-a parecer natural [...]. (Bourdieu, 1990, p. 34). 
(Berger; Luckman, 1973; Schutz, 1979; Garfinkel, 1984; Goffman,1980; 1985; Becker, 2008).

Durante os anos noventa e a primeira década do novo milênio, estudos em sociologia e antropologia, inserindo identidades, ações e representações sociais de pessoas e grupos na cultura global, multiplicaram-se, envolvendo frequentemente abordagem multidisciplinar.

É lícito, desse modo, pensar na superação da dicotomia paradigmática do fim do século XX, que engessava a produção científica da área, ao antepor abordagens metodológicas e teóricas aos objetos em construção e ao propiciar modos de apreensão dos fatos sociais pouco conectados com sua complexidade e mutabilidade histórica, embora permaneçam, tanto nos estudos disciplinares como na formação acadêmica, sobretudo pós-graduada, traços da histórica separação teoria-pesquisa empírica. Mas a convivência multi e interdisciplinar na área, assim como crescentes pesquisas intercampos disciplinares, (saúde e ciências humanas, por exemplo) tendem, a nosso ver, a minimizar esta permanência.

Nossa abordagem disciplinar do universo simbólico contemporâneo, ao pretender-se fronteiriça entre a antropologia social, a sociologia da cultura e a comunicação social, tem do universo cultural a visão de um todo, marcado ao mesmo tempo por enorme complexidade e fragmentação, e pela coexistência - frequentemente híbrida - de diversidade cultural inédita na história. Buscamos lançar alguns interrogantes sobre o imaginário social presente neste todo fragmentário, impregnado da racionalidade científica, no qual os significados e os sentidos sociais atribuídos a todos os aspectos do viver, em termos individuais ou coletivos, são constantemente invadidos por imagens, representações, modos de sentir, agir e avaliar racionalizados, isto é, imbuídos de sentidos, concepções e ideologias transmitidos e reproduzidos por saber(es) científico(s), através da divulgação cotidiana de suas últimas - ainda que provisórias - verdades 
pelos velozes meios de informação e comunicação atuais, ou, em linguagem comum, pelo conjunto da mídia, grande criadora e reprodutora de padrões culturais na sociedade contemporânea.

Os temas da cultura de comunicação de massa e dos veículos de divulgação que a expressam (mass media) começaram a ter importância nas ciências sociais no final dos anos 40, no período pós-guerra, nos Estados Unidos, com estudos sociológicos como o do cientista social David Riesman, A multidão solitária, lançado em inglês em 1950 (The lonely crowd), traduzido no Brasil quase três décadas após (Riesman, 1995), e Rostos na Multidão (Faces in the crowd), publicado em inglês em 1952.

Nas décadas seguintes, desencadeou-se no mundo ocidental um conjunto de pesquisas empíricas e de interpretações teóricas sobre o fenômeno social da mídia - concernindo, então, a comunicação impressa, isto é, jornais e revistas, e o rádio e a televisão - denominada MCM (meios de comunicação de massa) na cultura contemporânea. Tais estudos e pesquisas deram origem à complexa área dos saberes disciplinares no campo da Comunicação Social. A mídia atual inclui os novos veículos de transmissão de informações, sobretudo os virtuais, de peso dominante no processo da comunicação.

A hipótese interpretativa deste trabalho, dando sequência a uma tradição de pensamento em sociologia da cultura elaborada a partir de clássicos como Max Weber - posteriormente desenvolvida por seguidores, como Pierre Bourdieu (1989), para mencionar embasamento preferencial - é que a ciência tende a funcionar, na contemporaneidade, em termos de hegemonia (ou autoridade cultural, segundo Gramsci (1979), ou simbólica, segundo Bourdieu, de modo similar à teologia católica na Idade Média, e a organização social do sistema acadêmico universitário, que a traduz discursivamente, como sua escolástica. 
Com a expressão "de modo similar", queremos dizer que, em termos estruturais, os saberes tecnocientíficos tendem a constituir-se na mitologia social atual, isto é, no conjunto complexamente sistematizado, imbricado - e híbrido - de imagens, representações, atitudes, palavras, pensamentos e ações construídos e socialmente partilhados, que literalmente conferem sentido ao mundo e à própria subjetividade dos indivíduos (Foucault, 1966; Weber, 1971; Merton, 1973; Bourdieu, 1979; 2004).

Apesar de o "conjunto" de nossa análise ser apenas mais ou menos sistematizado, muitas vezes sincrético, bricolando simbolicamente saberes científicos e laicos, dispõe de modos de crença, de funcionamento, sobretudo de transmissão e convencimento, mais complexos que o das culturas pré-modernas, ou sem escritura, fundadas na transmissão oral, restringindo-se a aspectos estratégicos de reprodução da vida social, como os dos sistemas de parentesco (Lévi-Strauss, 2003). O conjunto cultural atual atinge todas as esferas do viver. Em outras palavras, na nossa cultura não há pensamento ou modo de agir aceito como racional - ou lógica considerada válida - que não seja derivado da ciência, ou que nela não encontre raízes de fundamentação. A ciência domina a racionalidade e se valida como modo correto do pensar e do agir das pessoas na sociedade.

Existem, certamente, outros campos culturais de produção de saberespráticas socialmente apreciados como modos de expressão de atividades, legitimados como portadores de sentidos e significados coletivos, como o artístico, o esportivo, o religioso e o próprio saber popular, dito laico, batizado também de senso comum, frequentemente associado à ignorância, à superstição ou mesmo à crendice. Sobre esses campos não se pode aplicar o timbre de portador de verdade, no sentido de fundamentado.

Não é do interesse deste trabalho desenvolver o tema do "desencantamento do mundo" ou da desmitologização produzida pela racionalidade cientifica na história ocidental moderna, tema explorado por Weber e 
por seus posteriores. O que desejamos problematizar aqui, inversamente, é a 'mitologização' da ciência na cultura contemporânea. Ou ao menos apontar para sua "utopização" (Sfez, 1995) através da análise da divulgação contínua de resultados de pesquisa, veiculados em revistas de divulgação científica, que apresentam um teor normativo de verdades saudáveis a serem incorporadas ao cotidiano de cada um, vista sua expressão discursiva racional técnica, irredutível ao mito, narrativa centrada no discurso simbóliCo, assim como ao rito, prática coletiva irredutível à racionalidade, embora seja portadora de significados que têm sua própria racionalidade.

É de outra racionalidade que se fala, nesse caso. A obra de alguns clássicos já nos envia para a questão da sistematização do pensamento mágico ou mitológico como conjunto de classificações e experiências paralelo ao científico ${ }^{4}$, ou mesmo do estabelecimento de práticas discursivas que formariam um conjunto de saberes e dispositivos fundadores de uma época com sua racionalidade própria5 ${ }^{5}$ (Foucault, 1994; Weber, 1995; Lévi-Strauss, 2003; Rabinow, 1999).

Interessa-nos, neste trabalho, problematizar, sobretudo, o processo cultural em andamento de transformação da ciência em utopia (Sfez, 1995, Luz 2008), analisando o papel estratégico que podem ter nesse processo as revistas de divulgação científica: papel de convencimento, de

\footnotetext{
${ }^{4}$ Lévi-Strauss (2003, p. 28), por exemplo, escreve: Em lugar de opor magia e ciência, seria melhor colocá-las em paralelo, como dois modos de conhecimento desiguais quanto aos resultados teóricos e práticos (pois, desse ponto de vista, é verdade que a ciência se sai melhor que a magia, no sentido de que algumas vezes ela também tem êxito), mas não devido à espécie de operações mentais que ambas supõem e que diferem menos na natureza que na função dos tipos de fenômeno aos quais são aplicadas.

${ }^{5}$ Foucault (1994) escreve que a ciência se elabora sob a coerção de uma instituição, a da pesquisa universitária, e sob a regra de se conformar a certo programa de rigor, sob pena de passar por não dizer o verdadeiro. Ela repousa sob um dispositivo feito de regras, tradições, ensinamentos, poderes, saberes, etc., que consagra e perpetua sua receita (a da ciência), regras de formação de enunciados aceitos como cientificamente verdadeiros, o "jogo de verdade" científico (Foucault, 1994, p. 58). Para uma abordagem mais aprofundada sobre estas questões no pensamento de Foucault, ver: Veyne (2011).
} 
persuasão das proposições científicas, não apenas como verdades intelectuais, mas também como preceitos morais, como guia de conduta para a vida como uma vida sadia. A ciência se apresentaria ao mesmo tempo como verdade a ser seguida, como doutrina intelectual e como prática litúrgica cotidiana. Esta é uma interpretação hipotética, que necessitaria ser confirmada - ou não - pela investigação da mídia impressa.

O que Sfez (1995) propôs como paradigma de análise para as biociências e sua intervenção na ordem da vida acreditamos poder ser extensivo à totalidade dos conhecimentos gerados pelo atual modo de produção de conhecimento técnico científico, em todas as disciplinas, mesmo as básicas, em função das atividades decorrentes da sua racionalidade no pensar, planejar e intervir, transformando e reformando a vida ambiental, individual e social, características básicas da ciência contemporânea (Luz, 2008).

A prática da pesquisa moderna não é um "ato livre" de pesquisadores, como foi, no período clássico do século XVIII, o "livre pensar" de filósofos iluministas (sobretudo os libertinos). Ela é fruto da intervenção de um conjunto de interesses e de atores sociais, não necessariamente cientistas, atuando como parceiros - ou adversários - que determinam sua possibilidade de iniciar um projeto: Agências Financiadoras, com suas prioridades e agendas, Instituições a que se liga o pesquisador ou grupo de pesquisadores (Universidades, Institutos etc.), além dos interesses públicos, privados ou estatais - com crescente influência no conjunto do processo da pesquisa (movimentos sociais, organizações, empresas) ${ }^{6}$ (Latour, 1987; Foucault, 1994; Rabinow, 1999; Bourdieu, 2004).

\footnotetext{
${ }^{6}$ Atualmente um pesquisador não é apenas um inventor, um criador de novas proposições teóricas ou práticas, um inovador de modos de pensar, explicar ou transformar o mundo, como foram os renascentistas e os clássicos modernos. Ele é tudo isto ao mesmo tempo, mas é também administrador, gerente e contador de seu projeto, e o divulgador e o publicitário dos resultados das suas atividades, além de um "cavador" de recursos para a continuidade das mesmas, no sentido de assegurar sua sobrevivência, buscando captar a simpatia dos seus pares e do Estado para a relevância de sua pesquisa e de seu desenvolvimento dentro de
} 
Esses múltiplos atores determinam desde a escolha de um tema de pesquisa até o(s) produto(s) final(ais) que deverá apresentar um projeto específico, num período específico para o desenvolvimento do mesmo. Pode o pesquisador assegurar à sua prática um mínimo de liberdade de escolha de objetos de pesquisa, de objetivos, de metodologia, e mesmo de fruto de seu trabalho, ou em outras palavras, de neutralidade? Em suma: de objetividade?

Os saberes gerados pela ciência contemporânea - sejam eles os estritamente disciplinares, nas pesquisas ditas "de bancada", ou os mais tecnológicos - têm em comum um forte viés de intervenção e modificação da "lógica das coisas" (naturais ou sociais), de seu ordenamento (ou re-ordenamento) e produção (ou reprodução) habitual, sejam essas coisas vivas ou objetos inertes, num pragmatismo em que a neutralidade axiológica nem está em questão, pois se visa, com a tecnociência, à contínua evolução do desenvolvimento da sociedade que originou esta forma de produção de saberes, de coisas, de relações entre as coisas (mercadorias, geralmente) e as pessoas, isto é, o moderno capitalismo ocidental (Weber, 2004). Esse modo de produção de saberes, atualmente chegado ao nível de desenvolvimento mundial por sua plena internacionalização, atinge a tudo e todos, em situação prevista tanto por Weber como por Marx (1965), quando este afirmou ser a ciência a força produtiva mais importante do capitalismo, em sua Introdução Geral à Crítica da Economia Política. A integração entre ciência e tecnologia, e sua necessária intervenção, no sentido do desenvolvimento das questões ambientais, naturais, sociais

prazos, com frequência demasiado estritos para garantir a almejada qualidade do projeto para seu autor (ou autores, já que a maior parte das atividades de pesquisa é de natureza coletiva, operando em redes).Tudo isto num contexto social de arena de competitividade entre os pares de fazer inveja aos gladiadores romanos. Tratando-se geralmente de funcionários estatais ou de Fundações mistas, a recompensa de pesquisadores, em termos materiais e simbólicos, é bem modesta quando comparada a outras carreiras profissionais do Estado. 
e biopsíquicas do mundo atual, no sentido do seu "aperfeiçoamento", é um objetivo inquestionável entre os atores envolvidos no processo.

Desse ponto de vista, têm razão analistas da ciência quando assinalam não existir atualmente prática científica "pura", nem pesquisa tecnológica "desinteressada", mas apenas tecnociência, um processo social ativo de produção de verdades e do real (Canguilhem, 1988; Foucault, 1989; Rabinow, 1999; Latour, Op. Cit.; Bourdieu, Op. Cit. Jullien, 2009).

Podemos também afirmar que a ciência, mais que como utopia, pode ser vista, nesse sentido, como uma ideologia de nosso tempo, discordando nesse ponto de Sfez (1995), que situa as ciências, tomando como caso as biociências, como a utopia de nossa era.

Mencionamos aqui a questão da ciência como ideologia por dois motivos: em primeiro lugar, pelo convencimento sistemático implícito no processo da cadeia de transmissão ideológica por seus dispositivos (institucionais e discursivos); em segundo lugar, pela estrutura racional normativa dos significados transmitidos pela ideologia científica, que adquirem autoridade ética ao se afirmarem como verdadeiros, pela lógica da demonstração empírica, tornando-se inquestionáveis ao nível da conduta, uma vez que já foram comprovados cientificamente. As verdades científicas, diferentemente das verdades perenes da teologia medieval, não se legitimam como proposições verdadeiras ad aeternum, mas como verdades provisórias (temporárias) comprovadas pela perenidade do método científico. O elemento perene, epistemológico, nesse caso, pois ratifica as verdades afirmadas como tal, é o método científico (Luz, 1988).

O processo social de inserção nesta ordem epistêmica, iniciado e continuado através do dispositivo escolar, cada vez mais precoce e duradouro na vida dos indivíduos é, na verdade, um processo de construção subjetiva, de interiorização nos sujeitos da autoridade epistemológica da ciência (e dos campos científicos), que Bourdieu (1989) denominou dominação sim- 
bólica. É parte essencial, embora não única, do processo de constituição de subjetividades de indivíduos e grupos, e de práticas sociais, garantidor de hegemonia ao(s) saber(es) tecnocientíficos na cultura atual (Nietzsche, 1978; Bourdieu, Op. Cit.; Rabinow, 1996; Foucault, 1997).

O conjunto atual dos dispositivos culturais retransmissores da ciência e da tecnologia como verdades vai, entretanto, muito além da escola, enraizando-se fortemente nos meios de informação e de comunicação de massa, e mesmo na organização social do sistema de trabalho nas empresas (Luz, 2008), tornado hoje praticamente "trabalho intelectual", como já afirmara Gramsci (1979) na primeira metade do século passado.

Esse conjunto instrumental de dispositivos tem seus meios próprios de difusão e transmissão de verdades, assim como de formação de criadores e difusores de novas verdades. No caso em análise neste texto, o conjunto de dispositivos é organizado sobretudo pelas redes de difusão e meios de divulgação, tanto midiáticos como virtuais: escritos, falados, ou simplesmente visualizados, isto é, baseados apenas na exposição de imagens das "verdades". Estas são divulgadas com linguagens diferenciadas, para públicos diferenciados, com papéis e estatutos sociais diferenciados, atribuídos a atores criadores e difusores das verdades, situados em posições hierarquizadas, segundo o meio de transmissão ou divulgação das verdades disciplinares que transmitem e inserem no sistema social vigente de informações/divulgação, variando do mais esotérico ao mais exotérico.

A questão da divulgação científica e das formas de disseminação dos saberes tecno-científicos na sociedade necessita, deste modo, de uma inadiável análise, buscando apreender o papel da ciência na formação de subjetividades e do imaginário atual.

Entre o processo de produção do conhecimento científico e tecnológico, através da prática da pesquisa, e a interiorização dos resultados desses saberes pelo senso comum dos indivíduos, grupos e classes que 
compõem a sociedade, para seu agir e pensar cotidianos, há uma "cascata de transmissão" de discursos socialmente hierarquizados, que vão do artigo especializado (difusão científica, no sentido mais esotérico, reservado aos pares do(s) pesquisador(es) autor(es) em seu campo), à publicidade (difusão social no sentido mais exotérico), divulgada através da mídia escrita, falada, visual e virtual, geralmente com a forma da propaganda midiática, com finalidades comerciais, ou mesmo ideológicas.

Um conjunto estruturado de meios de divulgação, entre esses, os virtuais, em grau mais ou menos douto, intervém no processo de divulgação, através sobretudo dos sites e das redes sociais da internet (Baumgarten, 2005).

Todos esses veículos podem ter em comum o fato de divulgar fatos, descobertas, ou avanços científicos com objetivos de convencimento (com finalidades comerciais ou normativas) dos conteúdos divulgados. Outros podem ter a finalidade oposta: debater e questionar a veracidade de tais "avanços", denunciando seu caráter mercantil ou ideológico.

Em face das questões provocativas mencionadas acima, constitutivas do núcleo duro de nosso tema de discussão, e levando em consideração sua profundidade, assim como as questões "de fundo" do mesmo que, reconhecemos, são de uma complexidade que exige não apenas tempo de reflexão, mas também investigação empírica, queremos deixar assinaladas, à guisa de conclusão, algumas questões que constituem o núcleo central do objeto deste artigo:

a) A ciência vem se tornando, face ao seu caráter normativo, uma verdadeira teologia laica desde o século recém-findo, e se afirma como tal desde a segunda metade do século XIX. Dispõe da mesma autoridade epistemológica e moral que a teologia medieval, uma vez que a dissidência por parte de seus praticantes é vista e punida pelo establishment acadêmico com o estigma profissional, e o desviante termina por ser afastado 
do "território da ciência", sendo moral e profissionalmente "posto em exílio", isto é, sendo impedido ou obstaculizado de exercício e divulgação de seus estudos, tendo sua carreira interrompida.

Por outro lado, os emissores de discursos definidos como leigos, ligados a saberes tradicionais, como os não ocidentais ou indígenas, por exemplo, são reduzidos ao não saber científico, ao senso comum, à ignorância, quando não ao charlatanismo, sofrendo os rigores das normas jurídicas ligadas ao saber científico, que defende a legalidade da ciência no ensino, na pesquisa e na atividade profissional (Bourdieu, 2008).

Considerando essas características marcantes da ciência contemporânea, seria possível ainda considerar esse modo específico de produção de saber democrático? Em que medida se pode distinguir, e de que modo, tal saber do da Teologia Medieval, materializado na Escolástica? Apenas pelo(s) modo(s) de difusão de verdades e seu alcance quantitativo?

b) Do ponto de vista epistemológico, simbólico e político, o que pode representar um regime de produção de saberes incapaz de dialogar com outros saberes fortemente presentes numa cultura marcada pela complexidade e pela diversidade? Trata-se de um saber monológico? Estaríamos diante um modo de produção de conhecimento colonizador, isto é, enfeudado a uma estrutura social com características específicas de dominação sociocultural, como na Idade Média, com a Teologia católica? Quais as consequências sociopolíticas, a longo prazo, desse regime de produção discursiva? Alguns cientistas sociais vêm discutindo esta questão nas últimas décadas, como Latour e Woolgar (1986), Morin (2002a; 2002b), Santos (2002), dentre outros.

c) Teria a ciência contemporânea condições ou interesse, considerado seu caráter discursivo epistemológico, fundado no método específico que utiliza para sua produção de verdades, avesso à relativização face a outros universos discursivos, de ver-se como uma cultura entre culturas, 
face à enorme diversidade das micro culturas presentes na cultura contemporânea? Em outras palavras, pode esse modelo discursivo considerar outro tipo de discurso como legítimo, reconhecendo a diferença, a pluralidade e a diversidade de saberes portadores de (outras) verdades? Isto é, de reconhecer a alteridade discursiva?

Dizendo de outro modo, tende a tecnociência a tornar-se uma monocultura engessada e voltada para as necessidades de reprodução econômica, social e política da sociedade que a originou a partir do século XVII?

d) Teria chegado o momento de analisarmos cuidadosa e criteriosamente, do ponto de vista da sociologia política da cultura e do conhecimento, ou da comunicação social (ou da interface dessas disciplinas conjuntamente), os gêneros - ou conteúdos - de imagens, de emoções, de sentidos, de representações sociais e de concepções que as tecnociências divulgam e nos transmitem, desde nossa infância, cada vez mais precocemente, sobre nossos corpos, nossos sentimentos, nossas vidas como um todo, no seu início, desenvolvimento, transcurso e fim, e também sobre a sociedade, sobre o ambiente que nos cerca, sobretudo sobre nosso próprio modo de conhecer e de nos expressar? Levantamos essas questões, estratégicas para o aprofundamento de pesquisa e reflexão no texto, bases de nosso projeto de pesquisa, face ao otimismo científico em relação à tecnociência que floresce no mundo acadêmico, mesmo entre sociólogos do conhecimento.

e) Que papel simbólico, político, ideológico ou pedagógico (ou todos eles) para a reprodução desse saber vem tendo a denominada divulgação científica, através da crescente circulação de periódicos de grande divulgação, que mobiliza e tematiza incessantemente as pesquisas disciplinares, abrangendo projetos e descobertas científicas em praticamente todas as disciplinas, sobretudo nas ciências da vida, dirigindo seus resultados e propostas normativas ao público "educado" (com formação uni- 
versitária), ou ao grande público (ensino médio completo ou incompleto), visto nesse contexto como "massa"?

Estas são algumas das questões lançadas por esse texto, que certamente, mais do que reflexão sociológica, está na raiz de uma pesquisa pluricêntrica interdisciplinar em início.

\section{Referências}

BAUMGARTEN, Maíra. (Org.) Conhecimento e Redes: sociedade, política e inovação. Porto Alegre: UFRGS; ALAS, 2005.

BECKER, Howard. Outsiders: Estudos de Sociologia do desvio. Rio de Janeiro: Zahar, 2008.

BERGER, Peter; LUCKMAN, Thomas. A Construção Social da Realidade. Petrópolis: Vozes, 1973.

BOAS, Franz. Antropologia Cultural. Rio de Janeiro: Zahar, 2005.

BOURDIEU, Pierre. A Economia das Trocas Linguísticas. Brasília: Edusp, 2008.

BOURDIEU, Pierre. Homo Academicus. Paris: Minuit, 1992.

BOURDIEU, Pierre. La distinction. Paris: Minuit, 1979.

BOURDIEU, Pierre. La Domination Masculine. Actes de la Recherche en Scienses Sociales, n. 84, p. 2-34, set. 1990.

BOURDIEU, Pierre. O Poder Simbólico. Lisboa: DIFEL; Bertrand Brasil, 1989.

BOURDIEU, Pierre. Sobre a televisão. Rio de Janeiro: Zahar, 1997. 143 p.

BOURDIEU, Pierre.Os Usos Sociais da Ciência: Por uma sociologia clínica do campo científico. São Paulo: Ed. UNESP, 2004.

BOZON, Michael. Amor, Sexualidade e Relações Sociais na França Contemporânea. Estudos Feministas, v.3, n. 1, p. 122-135, 1995.

CANGUILHEM, Georges. Ideology and Racionality in the History of the Life Sciences. Cambridge: MIT Press, 1988.

CUCHE, Denys. A Noção de Cultura em Ciências Sociais. Bauru: Edusc, 1998.

DURKHEIM, Émile. As Regras do Método Sociológico. São Paulo: Companhia Editora Nacional, 1972.

FOUCAULT, Michel. Dits et Écrits. Paris: Galimard, 1994. v. 3 
FOUCAULT, Michel. Les Mots et les Choses: une archéologie des sciences humaines. Paris: Seuil, 1966.

FOUCAULT, Michel. Microfísica do Poder. Rio de Janeiro: Zahar, 1989.

FOUCAULT, Michel. Resumo dos Cursos do Collège de France (1970-1982). Rio de Janeiro: Jorge Zahar, 1997.

GARFINKEL, Harold. Studies in Ethnometodology. Cambridge: Polity Press, 1984.

GEERTZ, Clifford. The Interpretation of Cultures. New York: Basic Books, 1977. GOFFMAN, ERVING. A Representação do Eu na Vida Cotidiana. Petrópolis: Vozes, 1985.

GOFFMAN, ERVING. Estigma: Notas sobre a manipulação da identidade deteriorada. Rio de Janeiro: Zahar, 1980.

GRAMSCl, Antonio. Os Intelectuais e a Organização da Cultura. Rio de Janeiro: Civilização Brasileira, 1979.

JAVEAU, Claude. Leçons de Sociologie. Paris, Armand Colin, 2005.

JULLIEN, François. O Diálogo Entre As Culturas: Do universal ao multiculturalismo. Rio de Janeiro: Zahar, 2009.

LATOUR, Bruno. Jamais Fomos Modernos. São Paulo: Editora 3, 2009.

LATOUR, Bruno. Science in Action: How to follow scientists and engineers through society. Cambridge: Harvard University Press, 1987.

LATOUR, Bruno. WOOLGAR, Steve. Laboratory Life: The Construction of Sicentific Facts. Princeton: Princeton University Press, 1986.

LÉVI-STRAUSS, Claude. Antropologia Estrutural. São Paulo: Cosac Naify, 2003. LÉVI-STRAUSS, Claude. O Pensamento Selvagem. São Paulo: Papirus Editora, 2002.

LUZ, Madel Therezinha. As Instituições médicas no Brasil: instituições e estratégia de hegemonia. 3. ed. Rio de Janeiro: Graal, 1986.

LUZ, Madel Therezinha. Natural, Racional, Social: Razão Médica e Racionalidade Científica Moderna. Rio de Janeiro: CAMPUS, 1997.

LUZ, Madel Therezinha. Notas sobre a política de produtividade em pesquisa no Brasil: conseqüências para a vida acadêmica, a ética no trabalho e a saúde dos trabalhadores. Política e Sociedade: Revista de Sociologia Política. v. 7, n. 13, p. 205-228, out. 2008.

LUZ, Madel Therezinha. Prometeu acorrentado: análise sociológica da categoria 
produtividade e as condições atuais da vida acadêmica. Physis, v. 15, n. 1, p. 3957, jan./jun. 2005.

MARX, Karl. Introduction Génerale à la Critique de I'Économie Politique. In: Karl Marx. Oeuvres. Paris: Gallimard, 1965. p. 234-266.

MERTON, Robert. The Sociology of Science: Theoretical and empirical investigations. Chicago: Chicago University Press, 1973.

MORIN, Edgar. Ciência com Consciência. São Paulo: Bertrand Brasil, 2002a.

MORIN, Edgar. Problema Epistemológico da Complexidade. Lisboa: EuropaAmerica, 2002b.

NIETZSCHE, Friedrich. Sobre Verdade e Mentira no Sentido Extra Moral. In: OBRAS Incompletas.. São Paulo: Abril Cultural, 1978. (Coleção Os Pensadores)

RABINOW, Paul. Antropologia da Razão. Rio de Janeiro: Relume-Dumará, 1999.

RABINOW, Paul. Making PCR: A Story of Biotechnology. Chicago: University of Chicago Press, 1996.

SANTOS, Boaventura Sousa. Um Discurso sobre as Ciências. Porto: Afrontamento, 2002.

SCHUTZ, Alfred. Fenomenologia e Relações Sociais. Rio de Janeiro: Zahar, 1979.

SFEZ, Lucien. La santé parfaite: critique d'une nouvelle utopie. Paris: Seuil, 1995.

VEYNE, Paul. Foucault: Seu pensamento, sua pessoa. Rio de Janeiro: Civilização Brasileira, 2011.

WAGNER, Roy. The Invention of Culture. Chicago: Chicago University Press, 1981.

WEBER, Max. A Ética Protestante e o Espírito do Capitalismo. Tradução revisada por Antônio Flávio Pierucci. São Paulo: Companhia das Letras, 2004. 335 p.

WEBER, Max. Economia y Sociedad. México: Fondo de Cultura Economica, 1995.

WEBER, Max. Ensaios de Sociologia. Rio de Janeiro: Zahar Editores, 1971.

WEBER, Max. La Acción Social: Ensayos Metodológicos. Barcelona: Ediciones Península, 1984. 